\title{
A Critical Review of the Role of Indicators in Implementing the Sustainable Development Goals
}

\author{
Forthcoming in the Handbook of Sustainability Science (2017), Edited by Walter \\ Leal.
}

Simon Mair, Aled Jones, Jonathan Ward, Ian Christie, Angela Druckman, and Fergus Lyon

\begin{abstract}
The 17 Sustainable Development Goals (SDGs) bring together environmental, social, and economic concerns. They therefore have the potential to move society away from the dominant model of prosperity as purely economic toward a more holistic and 'sustainable' prosperity. The success of such a transformative agenda rests on its implementation. At the heart of planned implementation of the SDGs is a set of 242 indicators. Indicators have been strongly critiqued in a range of literatures. However, in the context of the SDGs, indicators have been described as 'essential' with little critical assessment of their role in implementation.

Therefore, this chapter aims to provide this critical voice. To do this, the chapter reviews critiques of indicators from sustainability science, anthropology and sociology and provides illustrative cases of indicators implementation. From this review we are able to draw lessons for the use of indicators in SDG implementation. Specifically, the chapter argues that indicators are reductionist and struggle with contested concepts. Nevertheless, by making the operationalisation of concepts visible and enabling quantified analysis, indicators can have a useful role in SDG implementation. However, this requires that indicator critiques are taken seriously and inform indicator use.
\end{abstract}

\section{Authors}

Corresponding Author: Dr Simon Mair,

Centre for Environment and Sustainability, University of Surrey, Guildford, UK s.mair@surrey.ac.uk

Phone: +441483684314

Professor Aled Jones

Global Sustainability Institute, Anglia Ruskin University, UK

aled.jones@anglia.ac.uk

Dr Jonathan Ward

School of Media and Communication, University of Leeds, UK

j.ward1@leeds.ac.uk

Mr Ian Christie

Centre for Environment and Sustainability, University of Surrey, Guildford, UK

i.christie@surrey.ac.uk

Professor Angela Druckman

Centre for Environment and Sustainability, University of Surrey, Guildford, UK

a.druckman@surrey.ac.uk 
Professor Fergus Lyon

Centre for Enterprise and Economic Development Research, Middlesex University, UK f.lyon@mdx.ac.uk

\section{Introduction}

The Sustainable Development Goals (SDGs) have 242 quantified indicators, described as essential for "the measurement of progress", ensuring "that no one is left behind", and "key to decision making" (UN, 2015, p. 12). While indicators are widely perceived as useful for understanding and communicating about sustainability, there is a substantial body of interdisciplinary work that is highly critical of indicators (e.g. Bell and Morse, 2008; Merry 2011). This critical voice is missing from SDG indicator discussions. Therefore this chapter reviews the literature that is critical of indicators and applies it in the context of the SDGs. In doing so we aim to 1) highlight potential pitfalls of using indicators in SDG implementation and therefore 2) develop our understanding of how indicators could be successfully used in implementation of the SDGs.

Before continuing with the chapter, it is worth outlining the limitations and constraints of this chapter. The chapter is based on a review of the literature that critically assesses indicators. This literature is voluminous, and we do not attempt here to cover it in its entirety. Instead we draw our theoretical arguments from several key texts and highlight specific cases using illustrative case studies.

\subsection{The Pros and Cons of Indicator Use}

Indicators are central to many sustainability initiatives because they are a useful way to generate knowledge of, and communicate about, complex issues. Indicators break complex issues into more readily understood chunks of information thus allowing communication between experts and non-experts (Merry, 2011, Morse, 2016). Likewise, through selecting and measuring a finite set of quantified indicators that approximate the essential elements of a concept, experts can 'measure' an otherwise immeasurable entity (Turnhout et al., 2007). There is a long history of using indicators in this way in the biological sciences, particularly ecology (Bell and Morse, 2008) and indicators are applied similarly in sustainability research. For instance, Mair et al. (2016) use four indicators (carbon emissions, employment, income, and profit) to frame their investigation into the sustainability of Western European clothing consumption. Using the four indicators, they examine how the sustainability of the Western European clothing supply chain has changed over time and identify the mechanisms behind these changes. In such cases, indicators can function as an analytical structure, mediating between the nuanced, complex, and difficult to interrogate concept of sustainability, and the blunt analytical tools with which complex systems can be investigated.

However, indicators have been widely critiqued. Indicators are reductionist analytical tools and their use risks oversimplification, particularly in highly complex and contested contexts (Bell and Morse, 2008, Merry, 2011). This can be especially problematic because of the ways in which users interact with indicators. Indicators are often assumed to be objective and complete descriptions of the concepts they measure, but in reality are often value-laden and incomplete (Merry, 2011, Porter, 1995). Those with different agendas may therefore exert power and lobby to ensure indicators do not challenge their interests. Moreover, indicators help us to construct knowledge and guide decision making. Consequently, where they inadequately describe a contested concept, that concept may even become re-defined in terms of its indicators (Espeland and Sauder, 2007). This can lead to policies and strategies that focus on 
what is measurable rather than addressing less tangible or measurable issues. For example, the use of GDP as an indicator of societal progress has led to a reframing of societal progress as predominantly about increased productive capacity of the economy, creating a 'growth imperative' (Jackson, 2016).

GDP is a particularly pertinent example of the dangers of indicators. First it is an inadequate measure of societal progress because it misses important factors that contribute to broader conceptions of progress (Anderson, 2014). Second, GDP growth is strongly correlated with negative environmental impacts, and the extent to which it can solve social problems is questionable (Victor, 2007). Moreover, although such critques occasionally find a home in political discourse (e.g. Kennedy, 1968, DETR, 1999) policymakers have yet to move on from the use of GDP as a proxy measure of progress, and of growth in GDP as the principal objective of most government policy (Victor, 2007).

\subsection{Indicators for a Better Future?}

Given their prominent place in the SDGs, this chapter engages with the ways in which indicators may be used to help imagine, explore and create a better future. The SDGs aim to bring about a world in which "all human beings can enjoy prosperous and fulfilling lives and that economic, social and technological progress occurs in harmony with nature." (UN, 2015, p. 2). However, such a vision is highly contested and (given that indicators can be problematic in the absence of a fully agreed definition) the use of indicators in the SDGs should be approached with care.

Therefore, in this chapter we critically engage with indicators, particularly where they have been used in the context of contested and complex phenomena. Based on a review of the sociological, anthropological and sustainability literatures, we critique indicators as they have been used for various socio-cultural projects, with a view to understanding how they may be used in implementing the SDGs.

The rest of the chapter is structured as follows. In the next section we describe the contested nature of the SDGs. In section 3 we highlight that indicators of contested concepts are not neutral, but instead represent a particular perspective on an issue. We then demonstrate the difficulties this raises by comparing the SDG indicators to other Sustainable Development Indicators. In section 4 we elaborate on this, drawing on examples from the cultural projects to highlight how indicators of contested concepts risk oversimplification when faced with the complexity of moral problems. Finally, in section 5 we discuss the implications of these critiques for indicator use, arguing that although they should influence indicator use, they do not altogether negate the usefulness of indicators.

\section{Contested Concepts and the Sustainable Development Goals}

The idea of a set of 'Sustainable Development Goals' is highly contested 'sustainability', 'development', and even 'goals' all relate to issues that are subjective and politically sensitive. Sustainability deals with heavily value-laden issues including the proper relationship between society and the nature and from one generation to another. Likewise, 'development' is an ill-defined term. The SDGs succeed the Millennium Development Goals, which viewed development as a project to be pursued by poor countries, aided by richer countries. The SDGs, however, are referred to as 'universal' and therefore presumably have a very different view of development (UN, 2015). Finally, the nature of 'goals' is not clear cut, given different levels of investment in the vision of a particular goal, as well as considerable 
differences in social and economic power, goals that for some nations are easily realisable, will for others be purely aspirational. As a result, progress towards the Millennium Development Goals, for instance, was "far from uniform across the world" (UN, 2006).

As a result, the vision that drives the SDGs is highly contested. This can be illustrated by considering those elements that relate to physical realities, and are therefore nominally less subjective than purely social aspects. For example, the need to reduce damage to the environment. For some, this is rooted in the view that there are 'planetary boundaries' that humanity must avoid crossing in order to remain in a 'safe operating space'. While this idea is grounded in physical science (Steffen et al., 2015), planetary boundaries are constructed in such a way as to preserve current conditions. For example, a biodiversity 'limit' assumes that we value today's biodiversity more than historic or future biodiversity and does not account for the idea that a new biodiversity could flourish under the conditions of a changed environment. Furthermore, the impact of planetary limits on our ability to 'prosper' is highly contested. The 'strong' view of sustainability sees natural and man-made capital as complements, rather than substitutes, and therefore requires us to be cognizant of physical limits. On the other hand there is an alternative reading of the impact of planetary limits ('weak' sustainability) which views them as being of negligible importance in the construction of sustainable policies (Neumayer, 2003).

If such issues appear complex, things do not become clearer when we look at other aspects of the SDGs. As we will see, issues such as poverty, inequality and decent work are far from analytical: what counts as 'decent', for example? How do we measure 'inequality'? What do we mean by 'poverty'?

\section{Indicators are limited interpretations, not objective descriptions.}

Contested concepts defy the naïve understanding of indicators as measures of the essential components of a system. In the naïve view, researchers construct new information or communicate about a system by combining and interpreting multiple indicators (Bell and Morse, 2008, Figure 1). This understanding is applicable in objective and well understood systems. But contested concepts are characterised by multiple, conflicting ideas about the system works and an indicator set is only able to represent a subset of these understandings. Moreover, the differences in system understandings result in different indicators (Meadows, 1998, Davis et al., 2015). Therefore, an indicator of a contested system should not be understood as a piece of information about a system, but a piece of information reflecting how an individual or group conceptualises that system (Figure 2). 


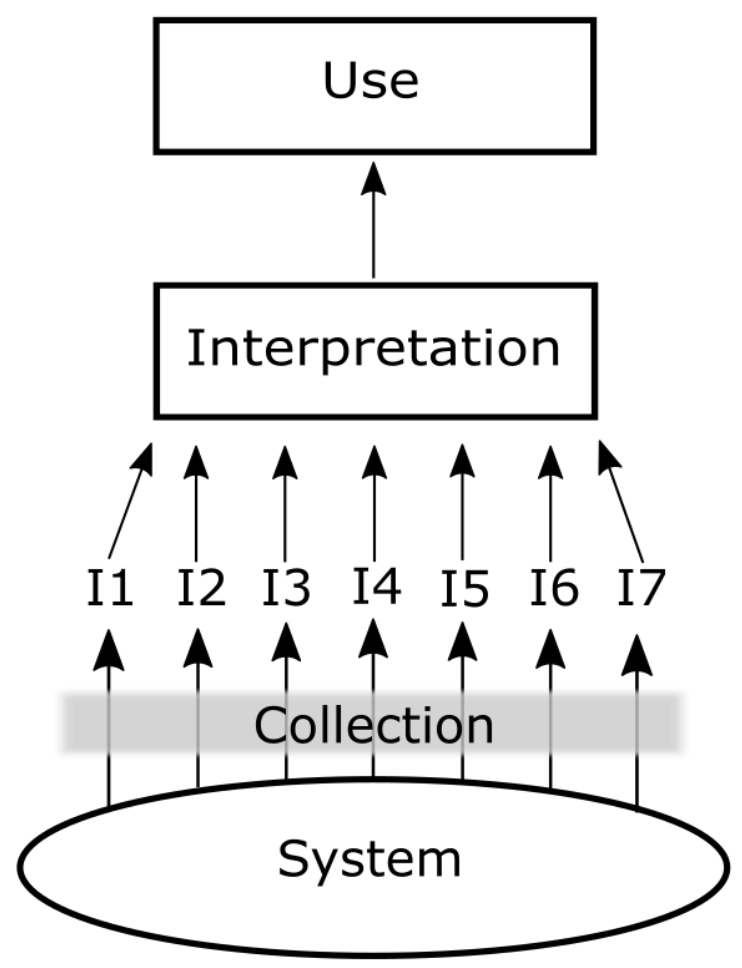

Figure 1 The naïve view of indicators (I), adapted from Bell and Morse (2008). I1 to I 7 collect information (indicated by arrows) about the system.

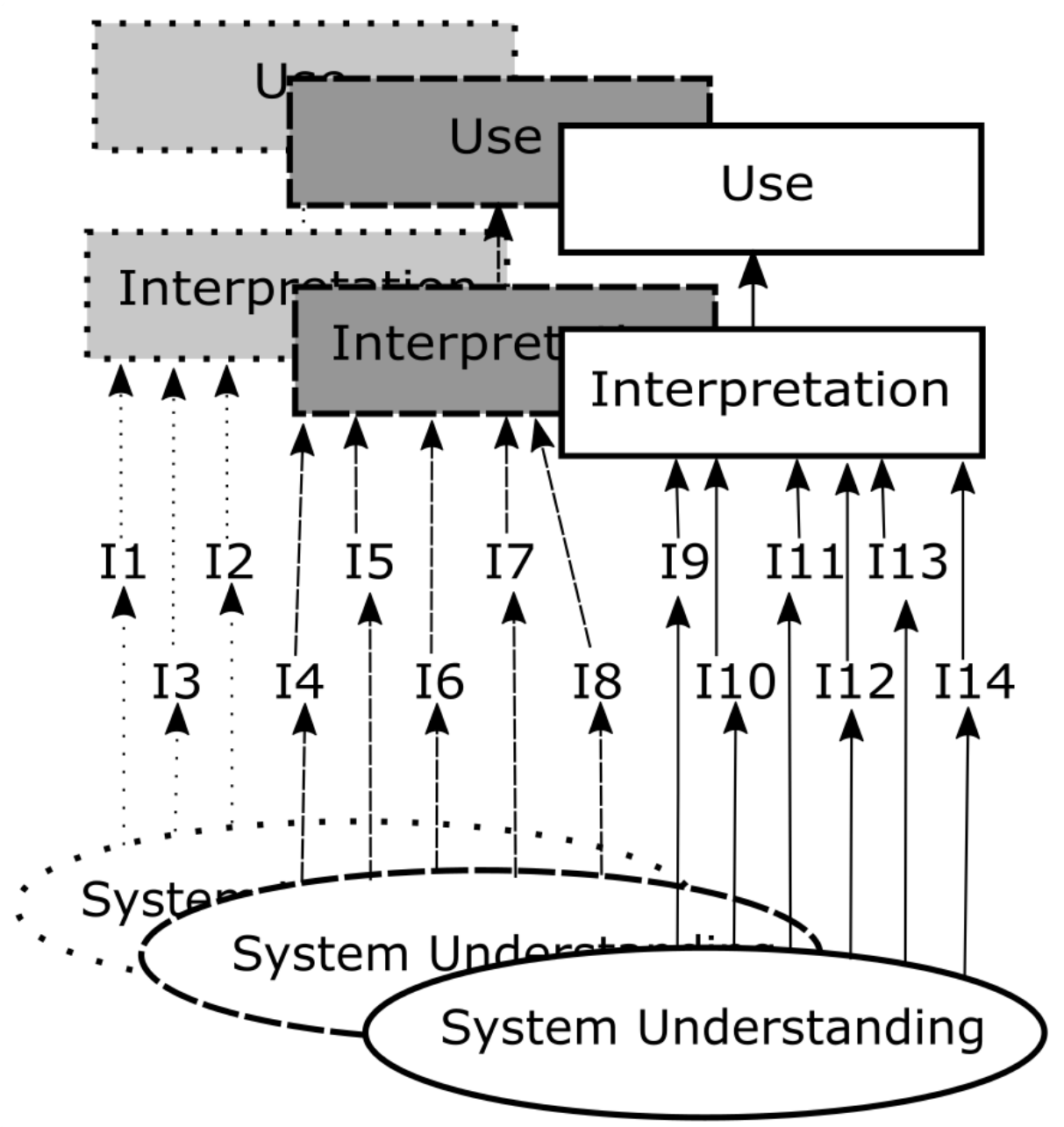

Figure 2 The concept of indicators as applied to highly contested and badly understood systems. While there are multiple conflicting understandings of contested systems, a given indicator set can only represent one (or a small subset) of those understandings. 


\subsection{Three different understandings of Sustainable Development}

To illustrate how the ability to represent only a limited perspective creates difficulties for indicators in contested systems, we compare and contrast the SDGs with two other sustainable development indicators sets. The UK (Lofts and Macrory, 2015) and the EU (Eurostat, 2015) have each developed a set of indicators that should be very similar to the SDG indicator set - all three indicator sets attempt to measure sustainable development and are primarily designed to be used by nation states.

However, there are substantial differences between each of the indicator sets. Not only can these indicator sets not agree on common statistical metrics - they fundamentally cannot agree on how to frame the indicator sets. At the most basic level, each set contains a different number of indicators or targets:

- SDGs - 17 goals, 169 targets, 242 Indicators;

- EU sustainable development indicators (SDIs) - 10 thematic areas, 132 indicators;

- UK SDIs - 3 thematic areas, 66 indicators.

Looking deeper, there are only three indicators that use the same statistical measure across the three frameworks: 1) GDP per capita, 2) Greenhouse gas emissions 3) Share of renewable energy. However, there are elements of each of these that are debated. There are only six further indicators that have a common outcome but differing statistical measures: 1) Increase research and development 2) Reduce air pollution (or the impact of air pollution) 3) Increase water efficiency 4) Increase river quality 5) Regulation of fishing 6) Protection of forests. Some of these differences are understandable, we would expect national and international frameworks to have some different targets (there is little reason for the UK to have a national target relating to rainforests, for example). However, many of the differences are less intuitive.

For example, 'poverty' appears in all three indicator sets, but each uses different statistical measures. The SDGs poverty indicators focus on the proportion of a national population suffering from various dimensions of poverty. Income and monetary aspects of poverty are captured through measures of people living below national and international poverty lines, while more social dimensions of poverty are captured by measuring the proportion of the population unable to access social protection systems and lacking access to basic services. In contrast, the UK's poverty SDIs focus predominantly on the proportion of children living in low income households, where income is defined both in absolute and relative terms.

Indicators both reflect and help construct theoretical perspectives and problem conceptualisations (Merry, 2011): the differences in the UK SDI and the SDG poverty indicators represent alternative understandings of poverty. The UK SDIs emerge from a conceptualisation of poverty as a primarily monetary problem, albeit with a role for societal norms around income. Further, the UK SDI theory of poverty sees households with children as the most at risk group (perhaps because they are most likely to be affected, or likely to suffer the most). Conversely, the SDG poverty indicators emerge from a conceptualisation of poverty as a problem that is broader than income alone (hence the inclusion of indicators on societal safety nets); they are based on a view of poverty as a problem for people of all ages (with indicators focused on a range of demographics); and they do not view societal norms as 
particularly important. Instead the SDGs focus on absolute understandings of poverty and have no explicit indicators for relative poverty.

3.2 Indicators shape how we view the world: alternative indictors can lead to conflicting outcomes.

The differences in the three indicator sets may even drive outcomes that undermine each other. Indicators refocus attention on to the elements of a concept that they measure and away from the elements that they do not. In this way, indicators direct how their users think about and attempt to deal with the concept itself (Espeland and Sauder, 2007, Merry, 2011). For example, the UK SDIs include a measure of the origin of food consumed in the UK, while the EU SDIs include a measure of imports from developing countries by group of products. Therefore, at the UK level there is a target to reduce food imports while at the EU level there is a target to increase them (albeit from a specific set of countries). Likewise, it seems reasonable to suggest that the UK SDI poverty indicators will result in different policies and outcomes than the SDG poverty indicators. For example, if the UK reduced the coverage of its social protection systems but simultaneously increased the average income of households with children, poverty could get both worse (according to the SDG definition) and better (according to the UK SDI definition)! 


\section{Indicators struggle with unquantifiable, moral issues.}

Indicators are further challenged by the difficulty of codifying, and linking important elements of contested concepts. The chief utility of indicators comes from the way they make complex issues more manageable through simplification. By definition, this requires losing information. Often, this information is the contextual and qualitative, discarded because it is hard to quantify, rather than because it is unimportant (Porter, 1995). Moreover, indicator sets have typically been developed without explicit consideration of their ethical basis or the moral assumptions embedded in their construction. Fredericks $(2014$, p. 6) argues that "there is a widespread assumption in modern Western society that technical and ethical assessments are, and should be, completely separable". This needs to be rejected, in her view, on the grounds that "developing indexes without explicit attention to ethics runs the risk of ineffective indexes, or even worse, indexes which drive people away from their vision of sustainability" (Fredericks, 2014, p. 9).

\subsection{Arts, Culture, and Ultimate Ends}

Informative examples of this crucial point about the ethical dimension of indicators, and the ways in which indicators struggle with difficult to codify ideas, are found in cultural indicator initiatives such as 'Taking Part'. Taking Part is perhaps the largest and most prominent attempt to provide data on the cultural sector in England. Research using Taking Part data claims to provide "robust" evidence (DCMS, 2014, p. 4) of links between certain kinds of cultural participation and wellbeing. However, establishing causal links between participation and other outcomes - such as increased wellbeing - proves difficult. Though quantitative analyses from the UK, as well as Canada, Italy and elsewhere, demonstrate a link between engagement in art and culture, and wellbeing, for many, "the challenges of disentangling confounding variables and establishing directions of causality remain" (Crossick and Kaszynska, 2016, p. 38).

Daly's Pyramid (Meadows, 1998; Figure 3), is a useful framework for understanding why connecting the Taking Part participation indicators to 'wellbeing' is difficult. Daly's Pyramid frames indicators as falling into one of four categories: at the top of the pyramid are Ultimate Ends - the things that we strive for, the high level concepts that together (arguably) constitute prosperity. At the base of the pyramid are the Ultimate Means - the fundamental earth systems without which we could not survive. In between the two are intermediate means (human labour, tools, etc.) which are used to produce intermediate ends (consumer goods, knowledge etc.). Intermediate ends are tools that are necessary to achieve our ultimate ends. 


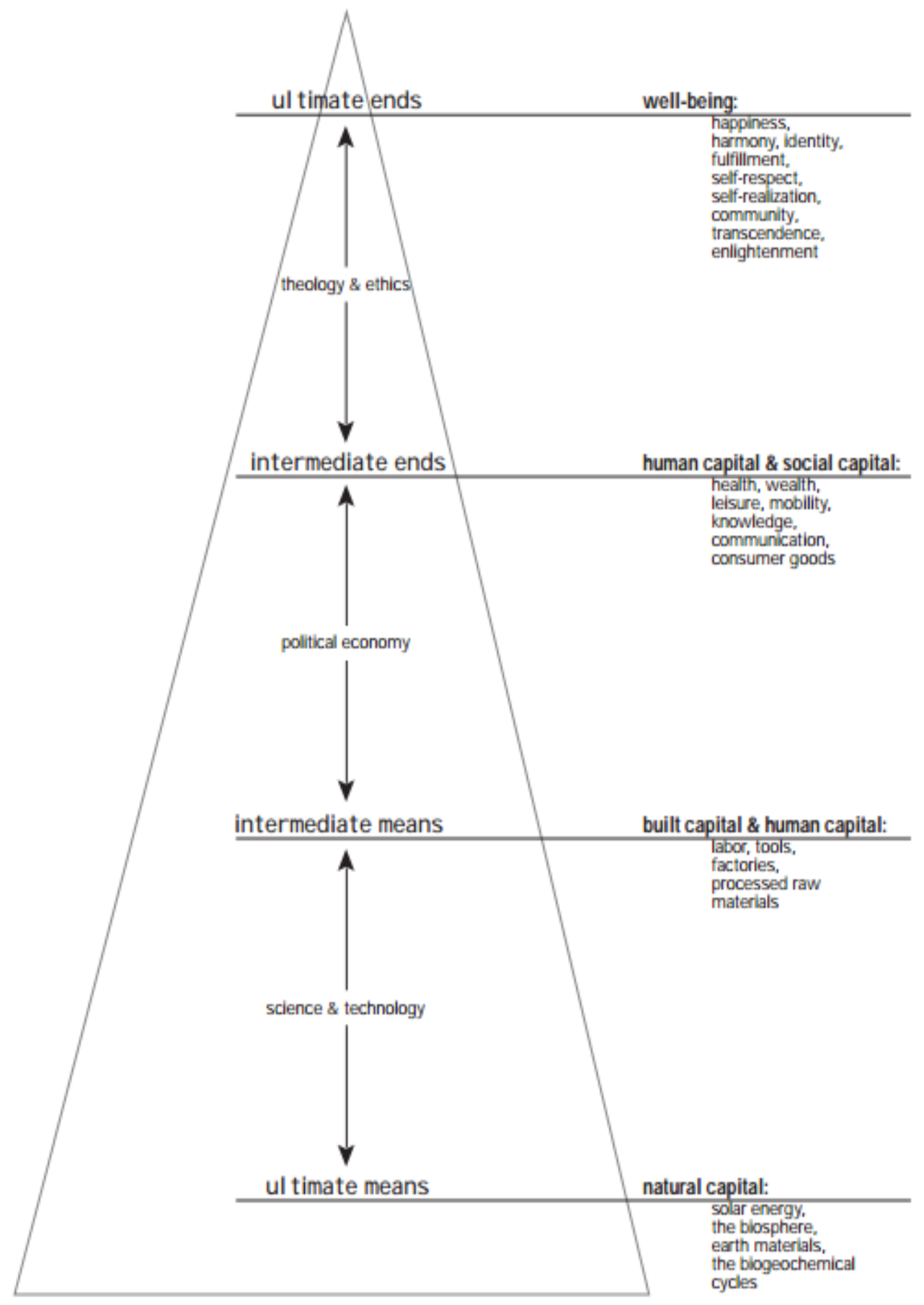

Figure 3 Daly's Pyramid, taken from Meadows (1998)

Taking Part measures intermediate ends, gathering information about participation and engagement in arts and cultural activities. This includes questions that aim to uncover drivers and barriers to participation as well as socio-demographic data. These data are released at 6 monthly intervals and provide headline figures on participation and engagement (DCMS, 2015).

However, 'wellbeing' is an ultimate end, not an intermediate end, and it is in connecting the two that problems arise. The data collected in Taking Part allows researchers to produce models that control for other factors (such as income) and provide a statistical evidence-base 
for claims about the positive impacts of cultural activity that speak to government objectives on wellbeing (Walmsley, 2012). But, as Daly's Pyramid makes explicit, ultimate ends indicators are the outputs of ethical and theological frameworks. In other words, they emerge from a process of highly personal interpretation informed not only by quantifiable measures but by emotional and moral reasoning. As Walmsley (2012, p. 329) argues, many of the ways that culture and art influence wellbeing are personal and intrinsic, taking us "into the immeasurable realms of spirituality and emotion".

It is also worth noting that lack of clarity and difficulty of quantification is not confined to the moral aspects of ultimate ends. It takes considerable effort "to arrange an unruly humanity into uncomfortable categories" (Porter, 1995, p. 41). Consequently, arbitrary exclusion and subjective categorisation are apparent even in the more mundane aspects of intermediate and ultimate ends indicators. Efforts to produce indicators for cultural work, for instance, are hampered by unclear boundaries and distinctions that make even counting the number of cultural workers difficult. Taking a sectoral approach to these labour markets includes large numbers of individuals in non- 'creative' roles while excluding cultural/creative workers in non-'creative' industries, while approaches that seek to utilise 'creative intensity' ultimately include roles that have little cultural output (Bakhshi et al., 2013).

\subsection{Indicators that ignore essential elements risk undermining the concept they purport to measure.}

These issues are particularly problematic because of the power of indicators to shape the thoughts and actions of researchers and decision makers: through indicator use, the more complex and qualitative aspects of ultimate ends risk being lost or ignored. As discussed in 3.2 , indicators can come to redefine concepts by directing attention only to those dimensions captured by the indicator (Merry, 2011). The act of measuring is not passive; rather, it shapes and defines what it is we are measuring, highlighting aspects to be important and, by omission, defining those aspects that are not important. As a result, indicators that ignore important elements of a concept may lead to policies that either overlook or actively conflict with the original concept as it is more broadly understood. In the arts, for example, Oakley et al. (2013, p. 24) point out that 'well-being-friendly' cultural policy may exacerbate current wellbeing inequalities.

Furthermore, there is a risk of concepts being redefined at all levels of society. Quantitative measures give the appearance of objectivity and neutrality: numbers often hide the complexity and value-laden nature of the judgements used in their construction (Porter, 1995). Where these constructions are very complex it is difficult for non-experts to challenge the indicator (Merry, 2011). Even where experts are willing to challenge the indicator, if the only dissenting voices have little political power it is easy for the indicator to remain neutral in appearance, and the concept to be re-defined (Espeland and Sauder, 2007).

Arts and culture once again provides a useful example of such risks. Neither art nor culture are included in the UK Office for National Statistics assessment of wellbeing, perhaps because they are too difficult to measure (Walmsley, 2012). Likewise there are no arts or culture based indicators under SDG goal 3 "Ensure healthy lives and promote well-being for all at all ages". Instead this goal opts for indicators that are based on much more easily quantified aspects of health such as maternal death rates and rates of new HIV infections. By ignoring arts and culture, wellbeing indicators may reduce actions that promote cultural and artistic dimensions of wellbeing; potentially leading to reduced wellbeing. 
Such issues are, however, not specific to wellbeing and the arts but also to other 'ultimate ends' central to the SDGs: community, harmony, fulfillment. How can personal growth, helping others or creating something new be measured? There may be proxies for wider social benefits, (such as health outcomes), but there is also a risk that inputs which are easier to measure (e.g. spending on healthcare) do not capture the desired outcomes and, if used as proxy indicators, become the desired outcomes in themselves.

\section{Can indicators be useful in implementing the SDGs?}

Despite the problems of indicators described in the above sections, we believe that indicators do have a useful role to play in implementing the SDGs. The previous two sections critiqued the use of indicators in contested concepts on the grounds that they are only able to represent a small subset of understandings of that concept, and that they struggle to deal with messiness and complexity. Here we draw on these critiques and begin to outline how we see indicators being usefully applied going forwards.

\subsection{Indicators can clarify political views and increase accountability.}

While indicators remove contextual information and obscure the process through which this happens, they also force a clarity and rigour that exposes political priorities and beliefs. This is seen clearly in the SDGs which often describe quite broad concepts that are accepted by a majority of global society and are apparently compatible with national sustainability initiatives. However, the SDG indicators reveal very specific perspectives on these problems some of which directly conflict with national perspectives. We have already discussed how indicators reveal very different conceptualisations of 'poverty' in the UK SDIs and the SDG indicators but this is not the only example of this in the SDGs.

Goal 8 aims to promote "decent work for all", a laudable purpose that few would disagree with. However, the SDG indicators have been criticised for failing to fully encapsulate the concept of decent work (Frey and MacNaughton, 2016). The SDGs do not, for example, include any measure of trade union coverage, working poverty rates or working time, all of which are 'main indicators' for decent work according to the International Labour Organisation (ILO, 2016). Moreover, there are no indicators of job satisfaction or fulfilment, reflecting a very different idea of 'decent work' than other where such ideas are central (see Burchell et al., 2014 for examples). These differences only become apparent in the indicators, because even the targets within Goal 8 refer to the contested idea of 'decent work', without making explicit how it is viewed.

This process of making a particular view explicit is, of course, the very same source of difficulty that we discussed in Section 3, here reframed as a strength. That indicators represent only a single perspective is a problem where they are interpreted as neutral fact, but a strength if indicators are understood as a clarification of a worldview. We must be clear here that indicators are no panacea, they do obscure those political judgments made in the construction of the indicator, but they also allow outsiders to see how concepts are being operationalised. So, instead of indicators necessarily re-conceptualising a problem and enforcing a single narrow view, they can also create a platform for debate and critique of a concept.

In part this is related to the public nature of indicators (Porter, 1995). Examining the decision making processes of the European Union (EU) and the Millennium Challenge Corporation (MCC), Dutta (2015) finds that the use of indicators makes those parts of the MCC decision making process that use indicators relatively transparent because, 
"external observers can more easily identify the mechanisms by which decisions are supposed to be made. Such legibility makes a contribution to accountability; where observers can easily discern how a decision was supposed to be made, they can more easily identify deviations in how the decision was actually made." (Dutta, 2015, p. 162)

Similarly, Finnerty (2005), argues that the use of indicators in the Irish National Anti-Poverty Strategy (NAPS) allows the government to be held to account and has formed the basis for much of the critique of the program.

Indicators may, then, be usefully used as a way of articulating the political vision that underlies the SDGs. In this way, indicators will facilitate external actors in both critiquing the goals and holding their governments to account. As we have seen, such a process has already begun in the academic literature (Frey and MacNaughton, 2016). We see no reason, however, why indicators should not be used to engage a much wider audience in conversations about the SDG program, how best to implement it and where it can be strengthened. That said, for this to work there would need to be an almost unprecedented level of transparency in the public reporting and discussion of the SDG indicators.

\subsection{Indicators facilitate new understandings of complex systems.}

While indicators may lead us to re-conceptualise issues in ways that somehow lessen or reduce our understanding of an issue, they can also facilitate a helpful reconceptualisation of knowledge. For example, Porter (1995 p. 37) argues that the widespread use of quantified indicators helped to create the idea of society by reframing individual problems as societal:

"Indeed the concept of society was itself a part statistical construct. The regularities of crime and suicide announced in early investigations of 'moral statistics' could evidently not be attributed to the individual. So they became properties instead of 'society'... Similarly, people sometimes found themselves or people they met to be out of work before this had become a statistical phenomenon. The invention of crime rates in the 1830 s and unemployment rates around 1900 hinted at a different sort of phenomenon, a condition of society involving collective responsibility rather than an unfortunate or reprehensible condition of individual persons"

By reframing a concept in this way, indicators can help us to consider new options and ways of thinking. Indeed the SDGs have been called transformative because they represent a much broader and more holistic view of societal progress than the current focus on GDP alone (WWF, 2016). As a result, the SDG indicators may prove to be tools that can be usefully used to highlight progress or problems. But, such application of the indicators should not be an end point in itself. Instead, this kind of indicator led analysis should direct and inform a more detailed and context-sensitive analysis. Similar roles have been played by subjective wellbeing indicators, which often find substantial geographical variation in wellbeing. Though the measures themselves cannot explain underlying causes, they do highlight potential problems to be explored further (Seaford, 2013).

The actual process of selecting indicators can also help understanding. Unfortunately, the SDG indicators have already been selected. Nonetheless, it is worth noting that by engaging in participatory processes, different views can be considered and space can be created for the voices of those who might otherwise be excluded (Fraser et al., 2006, Bell and Morse, 2008). 
By understanding the contested nature of indicators, those indicators selected can be refined and the limitations of any research identified.

Finally, the SDG Indicators will also enable quantified forms of analysis that can enhance our understanding of how the broader SDG targets might be achieved. A key example of this, in our view, is in the use of models. The SDGs operate in highly complex, non-linear systems, and such systems are difficult to interrogate without models because multiple interlinkages and feedback mechanisms can result in counter-intuitive emergent behaviours (Sterman, 2000). Models can be viewed as tools that mediate between theory and reality, drawing from both theories and the 'real' world, but retaining some autonomy (Morgan and Mary, 1999). Therefore, models facilitate learning by allowing users to test and refine their theories, value judgments and assumptions. By manipulating models, we are able to see how the outputs of a model diverge (or converge), from the theoretical predictions or 'real world' observations and explore why this is the case. Moreover, where models are sufficiently representative of some aspect of the real world they can be considered 'surrogate' worlds and we can make qualified inferences from our model world to the real world (Mäki, 2009). Indicators are essential in this process because they provide the mechanism that allows the model world, theory and reality to be compared.

\section{Conclusion}

This chapter has argued that the use of quantified indicators in the Sustainable Development Goals (SDGs) is problematic because the SDGs are a collection of highly contested concepts. Drawing on critiques of indicators from the sociological, anthropological and sustainability literatures, we showed that the reductive nature of indicators can create problems as they try to simplify and codify complex and subjective issues like those found in the SDGs. Highlighting the case of poverty, it was demonstrated that the SDG indicators only represent a limited understanding of poverty, and one that clashes with national understandings. Building from this example, we argued that any given indicator set (including the SGD indicator set) should be understood as a necessarily incomplete and value-laden view of a concept. As a result, use of one set of indicators may undermine attempts to achieve the same notional objective measured using alternative indicators. Likewise, drawing on an example from a cultural indicator projects, we made the case that indicators often arbitrarily strip away relevant information because it is hard to formally codify. We stressed that this is problematic because indicators change how policymakers and other change agents interact with and think about concepts.

On the basis of these critiques we argued that use of indicators in implementation of the SDGs should be handled carefully, but that there are roles that the indicators can usefully play. The SDG indicators could facilitate new understandings of sustainability and new learning through quantified analysis (such as modelling). However, such work should be the starting point of an analysis, not the end point. We also argued that given a more appropriate interpretation of the SDG indicators, their weaknesses can be reframed as strengths.

Therefore, going forwards we recommend that researchers and practitioners understand SDG indicators as an expression of the political priorities of the SDGs. Framed in this way, we suggest that the SDG indicators be used as a tool to engage citizens in debates about the SDGs, both to increase buy in and as a platform for critique of the SDGs (as is happening with Goal 8 in the academic sphere). Likewise, we suggest that the SDG indicators might be used to hold governments to account, as has happened in other development initiatives. In summary, future work on the SDG indicators, (and indicators more widely) should be used to inform, engage 
and hold to account policy development processes. SDG indicators should not be the main basis on which to develop new policy.

\section{Acknowledgements}

This work was completed as part of the ESRC Research Centre for the Understanding of Sustainable Prosperity, grant number ES/M010163/1.

\section{References}

Anderson, A., (2014) Alternative Economic Indicators? UK and USA: Routledge, pp. 116.

Bakhshi, H., Freeman, A. and Higgs, P. 2013. A Dynamic Mapping of the UK'S Creative Industries. UK:NESTA, pp. 79.

Bell, S. and Morse, S. (2008) Sustainability indicators: measuring the immeasurable? UK and USA: Earthscan, pp. 251.

Burchell, B., Sehnbruch, K., Piasna, A. and Agloni, N. (2014) 'The quality of employment and decent work: definitions, methodologies, and ongoing debates', Cambridge journal of economics, 38(2), pp. 459-477

Crossick, G. and Kaszynska, P. (2016) Understanding the value of arts \& culture. Swindon: Arts \& Humanities Research Council. Available at: http://www.ahrc.ac.uk/documents/publications/cultural-value-project-final-report/ (Accessed: 11/10/2016).

Davis, K., Kingsbury, B. and Merry, S. (2015) 'The Local-Global Life of Indicators: Law, Power, and Resistance', in Merry, S., Davis, K. \& Kingsbury, B. (eds.) The Quiet Power of Indicators: Measuring Governance, Corruption, and Rule of Law USA: Cambridge University Press, pp. 1-26.

DCMS (2014) Culture, Sport and Wellbeing: An analysis of the Taking Part Survey. London: Department for Culture Media and Sport, pp. 13.

DCMS (2015) Taking Part 2015 / 16 Quarter 2. London: Department for Culture Media and Sport, pp. 69

DETR (1999) A Better Quality of Life: the UK Sustainable Development Strategy. London: Department for the Environment, Transport and the Regions,

Dutta, N. (2015) 'Tradeoffs in Accountability: Conditionality Processes in the European Union and Millennium Challenge Corporation', in Merry, S., Davis, K. \& Kingsbury, B. (eds.) The Quiet Power of Indicators: Measuring Governance, Corruption and the Rule of Law. USA: Cambridge University Press, pp. 156-196.

Espeland, W. and Sauder, M. (2007) 'Rankings and reactivity: How public measures recreate social worlds', American Journal of Sociology, 113(1), pp. 1-40.

Eurostat (2015) Sustainable development in the European Union: 2015 monitoring report of the EU Sustainable Development Strategy. Luxembourg: Eurostat Statistical Books, pp. 356.

Finnerty, J. (2005) 'Social Indicators: Pitfalls and Promise', in Herrman, P. (ed.) Utopia Between Corrupted Public Responsibility and Contested Modernisation: Globalization and Social Responsibility, USA: Nova Science, pp. 61-76.

Fraser, E., Dougill, A., Mabee, W., Reed, M. and McAlpine, P. (2006) 'Bottom up and top down: analysis of participatory processes for sustainability indicator identification as a pathway to community empowerment and sustainable environmental management', Journal of Environmental Management, 78 (2), pp., 78(2), pp. 114-127.

Fredericks, S. (2014) 'Measuring and Evaluating Sustainability: ethics in sustainability indexes'.USA and Canada: Routledge, pp. 230.

Frey, D. and MacNaughton, G. (2016) 'A Human Rights Lens on Full Employment and Decent Work in the 2030 Sustainable Development Agenda', SAGE Open, 6(2), pp. 1-13. 
ILO (2016) Decent Work: International Labour Organisation. Available at: http://www.ilo.org/global/topics/decent-work/lang--en/index.htm (Accessed: 01/08/2016).

Jackson, T. (2016) Prosperity without Growth - foundations for the economy of tomorrow. London and New York: Routledge, pp 336.

Kennedy, R. (1968) Remarks at the University of Kansas. Available at: http://www.jfklibrary.org/Research/Research-Aids/Ready-Reference/RFKSpeeches/Remarks-of-Robert-F-Kennedy-at-the-University-of-Kansas-March-18-1968.aspx (Accessed: 02/08/2016).

Lofts, H. and Macrory, I. (2015) Sustainable Development Indicators: July 2015 Office of National Statistics, UK. Available at: http://www.ons.gov.uk/peoplepopulationandcommunity/wellbeing/articles/sustainabledev elopmentindicators/2015-07-13 (Accessed: 29/07/2016).

Mair, S., Druckman, A. and Jackson, T. (2016) 'Global Inequities and Emissions in Western European Textile and Clothing Consumption', Journal of Cleaner Production, 132, pp. 57-69.

Mäki, U. (2009) 'MISSing the world. Models as isolations and credible surrogate systems', Erkenntnis, 70(1), pp. 29-43. .

Meadows, D. (1998) Indicators and Information Systems for Sustainable Development: A report to the Balaton Group. USA: The Sustainability Institute, pp. 95.

Merry, S. (2011) 'Measuring the World: Indicators, Human Rights, and Global Governance', Current Anthropology, 52(3), pp. S83-S95.

Morgan, M. and Mary, M. (1999) 'Models as Mediating Instruments', in Morgan, M. \& Mary, M. (eds.) Models as Mediators, UK: Cambridge University Press, pp. 10-37.

Morse, S. (2016) 'Measuring the success of sustainable development indices in terms of reporting by the global press', Social Indicators Research, 125, pp. 359-375.

Neumayer, E. (2003) Weak versus strong sustainability : exploring the limits of two opposing paradigms. Cheltenham: Edward Elgar, pp. 271.

Oakley, K., O'Brien, D. and Lee, D. (2013) 'Happy now? Well-being and cultural policy', Philosophy \& Public Policy Quarterly, 31(2), pp. 18-26.

Porter, T. (1995) Trust in Numbers: The Pursuit of Objectivity in Science and Public Life. Chichester: Princeton University Press, pp.310.

Seaford, C. (2013) 'The multiple uses of subjective well-being indicators', Social indicators research, 114(1), pp. 29-43.

Steffen, W., Richardson, K., Rockström, J., Cornell, S. E., Fetzer, I., Bennett, E. M., Biggs, R., Carpenter, S. R., de Vries, W., de Wit, C. A. and Folke, C. (2015) 'Planetary boundaries: Guiding human development on a changing planet', Science, 347(6223), pp. 125985.

Sterman, J. (2000) Business dynamics: systems thinking and modeling for a complex world. Boston: Irwin/McGraw-Hill, pp. 982.

Turnhout, E., Hisschemöller, M. and Eijsackers, H. (2007) 'Ecological indicators: between the two fires of science and policy ', Ecological Indicators, 7(2), pp. 215-228.

UN (2006) UN Millennium Goals, About the MDGs. Available at: http://www.unmillenniumproject.org/goals/ (Accessed: 16/09/2016).

UN (2015) Resolution adopted by the General Assembly on 25 September 2015, Transforming our world: the 2030 Agenda for Sustainable Development. Available at: https://sustainabledevelopment.un.org/post2015/transformingourworld (Accessed: 09/06/2016).

Victor, P. A. (2007) Managing Without Growth: Slower by Design, Not Disaster. Cheltenham UK: Edward Elgar Publishing, pp. 272.

Walmsley, B. (2012) 'Towards a balanced scorecard: A critical analysis of the Culture and Sport Evidence (CASE) programme', Cultural trends, 21(4), pp. 325-334. 
WWF (2016) Sustainable Development Goals. Available at:

http://www.wwf.org.uk/what_we_do/making_the_links/sustainable_development_goals/ (Accessed: 23/07/2016). 